\title{
Porphyrin-Based Nanostructures for Sensing Applications
}

\author{
Donato Monti, ${ }^{1}$ Sara Nardis, ${ }^{1}$ Manuela Stefanelli, ${ }^{1}$ Roberto Paolesse, ${ }^{1}$ Corrado Di Natale, ${ }^{2}$ \\ and Arnaldo D'Amico'
}

${ }^{1}$ Dipartimento di Scienze e Tecnologie Chimiche, Università di Roma “Tor Vergata”, Via della Ricerca Scientifica, 00133 Roma, Italy

${ }^{2}$ Dipartimento di Ingegneria Elettronica, Università di Roma “Tor Vergata”, Via del Politecnico, 00133 Roma, Italy

Correspondence should be addressed to Roberto Paolesse, roberto.paolesse@uniroma2.it

Received 14 February 2009; Revised 28 April 2009; Accepted 29 May 2009

Recommended by Yongxiang Li

\begin{abstract}
The construction of nanosized supramolecular hosts via self-assembly of molecular components is a fascinating field of research. Such intriguing class of architectures, beside their intrinsic intellectual stimuli, is of importance in many fields of chemistry and technology, such as material chemistry, catalysis, and sensor applications. Within this wide scenario, tailored solid films of porphyrin derivatives are structures of great potential for, among others, chemical sensor applications. The formation of supramolecules relays on noncovalent interactions (electrostatic, hydrogen bond, $\pi-\pi$, or coordinative interactions) driven by the chemical information stored on the assembling molecules, such as shape and functional groups. This allows, for example, the formation of large well-defined porphyrin aggregates in solution that can be spontaneously transferred onto a solid surface, so achieving a solid system with tailored features. These films have been used, covering the bridge between nanostructures and microsystems, for the construction of solid-state sensors for volatiles and metal ion recognition and detection. Moreover, the variation of peripheral substituents of porphyrins, such as, for example, chiral appended functionalities, can result in the formation of porphyrin aggregates featuring high supramolecular chirality. This would allow the achievement of porphyrin layers characterised by different chiroptical and molecular recognition properties.
\end{abstract}

Copyright ( 2009 Donato Monti et al. This is an open access article distributed under the Creative Commons Attribution License, which permits unrestricted use, distribution, and reproduction in any medium, provided the original work is properly cited.

\section{Introduction}

In the last few years the prefix nano- has been probably one of the most popular and widely used buzzwords, not only in the scientific community, but also to generally indicate systems whose components have dimensions in the nanometric scale. This general interest is due to the great expectation for the potential impact that the development of such miniaturized systems can have on fundamental topics, such as, for example, energy, environment, or security.

Despite this large popularity, the exact meaning of nanoscience and nanotechnology is not universally defined, as usual for a discipline not completely mature, anyhow the most general definition is related to the development and manipulation of structures (objects or devices) in the size range lower than $100 \mathrm{~nm} \mathrm{[1].}$

Even if this definition is not completely satisfying, one topic is for sure critical for nanotechnology: the miniaturization of the systems of interest is the necessary requirement to improve the performances of the related devices. The dramatic development of electronic devices is in fact directly related to the size reduction of their components.

Miniaturization has been usually obtained by reducing the dimension of bigger components using techniques such as photolithography; this method is called "top-down approach" and, although well developed, it reaches its limit at the open door of the nanotechnology domain [2].

To overcome these limitations, the natural solution is represented by the opposite approach, which has been called "bottom-up approach;" the devices are build up from their fundamental building blocks, atoms or molecules, by their rational assembling.

Since the report of the preparation of carbon nanotubes [3], we have witnessed the preparation of a wide range of different nanostructured inorganic materials, obtained by the fine control of the growth conditions of such materials [4]. 
In the case of organic compounds, which are constituted by molecules, the preparation of nanostructures is far less developed; while the synthesis of molecular systems is a well-developed discipline, driven by the formation of stable chemical bonds, nanostructures should derive from multiple, weak, and noncovalent interactions of different nature, such as electrostatic and van der Waals forces or hydrophobic effects, $\pi-\pi$ stacking interactions, metal coordination, and hydrogen bonding.

However, the ability to assemble molecular units in more complex architectures has taken advantage of the supramolecular chemistry development, the so-called chemistry beyond the molecule $[5,6]$. Capitalizing the plurality of weak interaction pathways, molecules can self assemble giving different nanostructure motifs, such as tubes, rods, and sheets [7].

Among the huge number of molecular systems suitable for such an approach, porphyrins represent one of the most promising examples, for different reasons. Their planar aromatic macrocycle is optimal for $\pi-\pi$ stacking interactions and it is a versatile platform for peripheral decoration with groups that can offer additional interaction motifs [8]. The synthetic chemistry of porphyrins is well developed and it is possible to choose among a wide substituent library to prepare the macrocycle of choice for the particular application $[9,10]$. All these features will not be sufficient to justify an interest in porphyrins, if we do not consider the richness of properties these macrocycles are endowed with [11]. Photophysical and coordination features are just an example of the potentialities of such a macrocycle. Nature offers a magnificent example of porphyrin usefulness, whereas with this basic molecular framework Nature is able to activate and/or transport molecular oxygen in animals and convert sunlight in plant photosynthetic systems [12].

All these properties can be transferred in nanostructured materials based on porphyrin derivatives, for the application in different fields, ranging from catalysis to pharmacology.

\section{Porphyrin-Based Nanostructures}

In the last few years the exploration of porphyrins, metalloporphyrins, and their assemblies afforded a large number of nanomaterials featuring various electronic and structural characteristics, like rods, rings, particles, sheets, wires, and tubes. We summarize below few examples of porphyrin nanostructures reported in the literature.

2.1. Porphyrin Nanoparticles. A first class of porphyrin aggregates is represented by the preparation of porphyrin nanoparticles, that is, colloidal suspension of porphyrin aggregates having dimension in the order of hundreds of $\mathrm{nm}$ [13]. The size dimension of the particles strongly depend on the porphyrin structure and on the preparative protocols, where it is usually used as a surfactant to stabilize the suspension, avoiding the collapse of the nanoparticles in larger aggregates.

The interest in these systems depends on the potential applications in different fields, such as catalysts, or drug delivery. The aim is to develop architectures that can offer a higher stability than monomer in solution, preserving or even increasing the activity of the starting molecular unit. The easiness of preparation and the possibility to modulate the size and properties of such nanoparticles represent the promising characteristics of these structures.

Drain et al., for example, exploited the formation of porphyrin nanoparticles by adding water to a solution of the porphyrin in an organic solvent, containing a few percent of a surfactant, such as low molecular weight poly-ethylenglycol [14]. The method works for a wide range of porphyrin structures; the presence of a surfactant is essential to assure the suspension stability, otherwise the porphyrin particles rapidly coagulate and precipitate from the solution. Following the same approach Sandanayaka et al. have characterized structural and photophysical properties of 5,10,15,20tetrakis(4-carboxyphenyl)porphyrin (Figure 1(a)) nanoparticles obtained using ethylene glycol and the solvent mixture technique [15].

A different approach was pursued by Bangal and Perepogu who recently prepared pure nanoparticles of $5,10,15,20$-tetraphenylporphyrin or 5,10,15,20-tetra(pentafluorophenyl)porphyrin (Figures 1(b)-1(c)), without the addition of surfactants as nanoparticle stabilizers [16]. In this case the approach is called "reprecipitation method" and it takes advantage of a careful choice of solvent mixtures, where the first one should act as good and the second as poor solvent to induce the nanoparticles formation.

2.2. Porphyrin Nanosheets. Wang et al. reported the preparation of porphyrin nanosheets by the reprecipitation method, injecting an ethanol solution of an SnPyTriPP complex (Figure 1(d)) into deionized water [17]. The formation of $2 \mathrm{D}$ aggregates are strongly dependent on the preparation method, because changing the water temperature led to completely different aggregates.

Following a different approach, large nanosheets have been prepared by Milic et al., by promoting dynamic aggregation in columnar stacks of square planar porphyrin nonamers, formed by TPyP units coordinated by $\mathrm{PdCl}_{2}$ bridging group through the peripheral N-py atoms [18].

2.3. Porphyrin Nanorods and Nanorings. 5,10,15,20-tetrakis(4-sulfonatophenyl)porphyrin (Figure 1(e)) self-assembles in acidic aqueous solution into rods that can span over $\mu \mathrm{m}$ lengths. These rods show an intriguing photoconductivity properties, with a rapid turn on/off rate $[19,20]$.

Nanorods are also obtained from the aggregation of 5,10,15,20-tetrakis(4-carboxyphenyl)porphyrin in acidic aqueous solutions [21]. In this case the structural motif of the aggregate strongly depends on the acid exploited for the aggregation; nanorods were obtained in $\mathrm{HNO}_{3}$ solutions, while $\mathrm{HCl}$ induced the formation of nanorings. Porphyrin nanorings have been also obtained by evaporation of the solvent through a surface dewetting process [22].

Supramolecular nanorods of 5,15-diaryl-substituted porphyrins have been prepared by sonication method from an acetonitrile/toluene solution, which represents a poor/good solvent mixture [23]. 


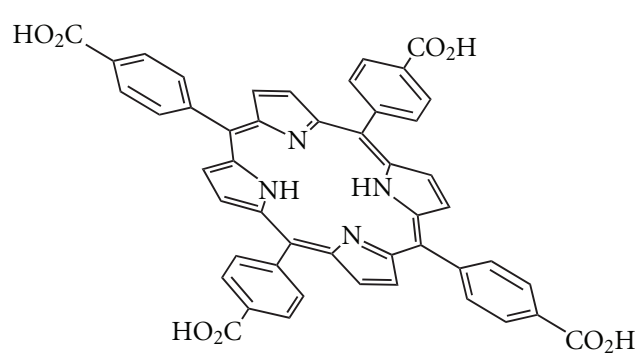

(a)

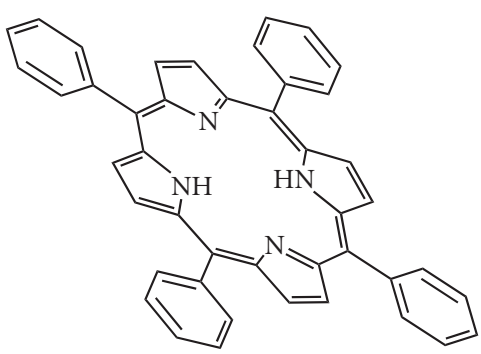

(b)

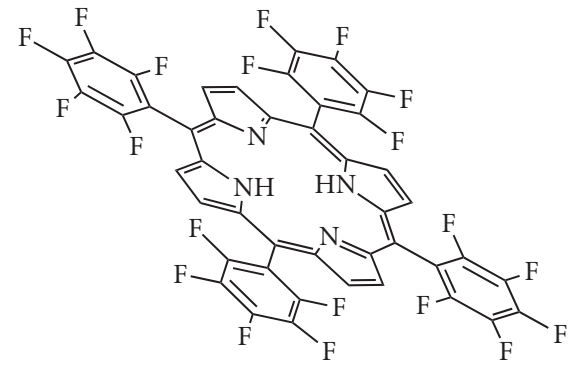

(c)

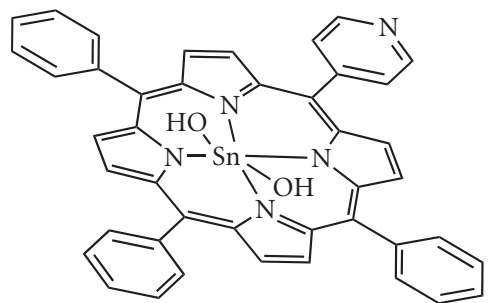

(d)

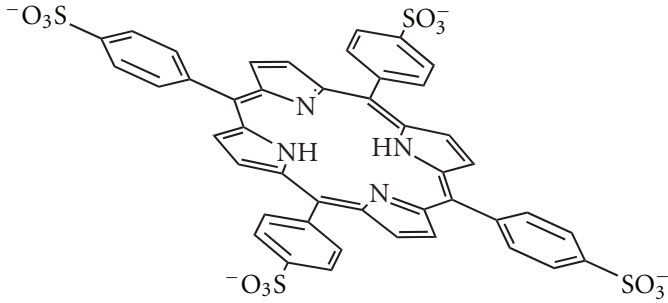

(e)

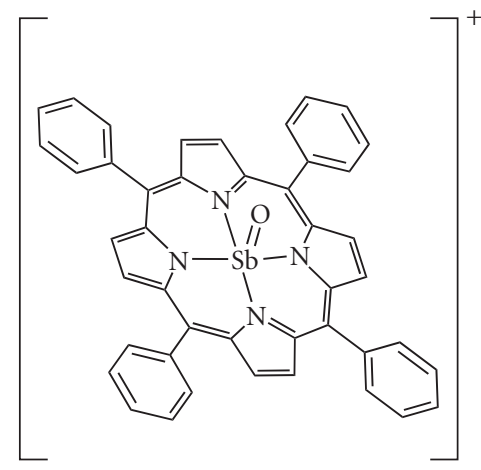

(f)

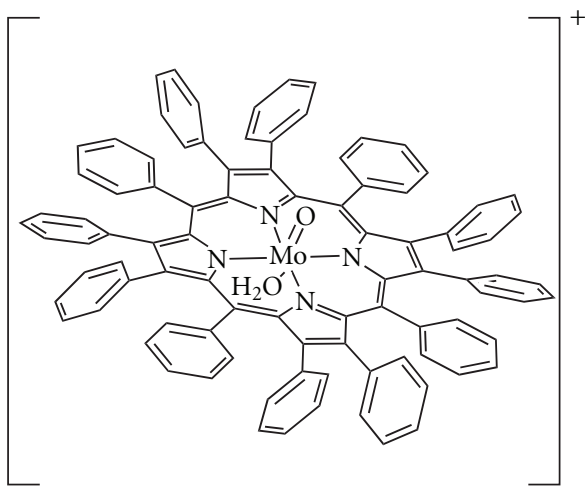

(g)

FIGURE 1: Molecular structures of (a) 5,10,15,20-tetrakis(4-carboxyphenyl)porphyrin; (b) 5,10,15,20-tetraphenylporphyrin; (c) 5,10,15,20-tetra(pentafluorophenyl)porphyrin; (d) 5-pyridyl-10,15,20-triphenylporphyrin Sn(IV) dihydroxide; (e) 5,10,15,20-tetrakis(4sulfonatophenyl)porphyrin; (f) cationic 5,10,15,20-tetraphenylporphyrin oxoSb(V); (g) cationic dodecaphenylporphyrin oxoMo(V).

Fullerene-encapsuled hexagonal nanorods of 5,10,15,20tetrakis(4-pyridyl)porphyrin $\mathrm{Zn}(\mathrm{ZnTPyP})$ have been prepared in DMF-acetonitrile solution with the aid of a surfactant and investigated to elucidate photoinduced electron transfer [24]. The nanostructure has a broad absorption spectrum, due to the supramolecular aggregation, and shows also a significant quenching of porphyrin fluorescence emission, in large part due to an efficient photoinduced electron transfer between ZnTPyP and fullerene. This behavior has been confirmed by comparison of the fluorescence of the empty ZnTPyP nanorods prepared without fullerene in the same conditions. Both the broad absorption spectrum and the presence of a photoinduced charge separation state $\left[\mathrm{C}_{60}{ }^{\circ-}-\mathrm{ZnTPyP}^{\circ+}\right]$ give a significant enhancement of the solar energy conversion, as demonstrated in a photoelectrochemical cell based on these nanorods as chromophores.

2.4. Porphyrin Nanowires. Dispersed nanowires can be obtained by assembly of amphiphilic Sn complex of porphyrin, whose lengths can be finely tuned using an amphiphilic polymer surfactant, Pluronic F127 [25]. The structural motif of the nanostructures ranges from nanowires to nanoplates by adjusting the synthetic protocol. The key factor is in this case the water solubility of the Sn porphyrin nanostructures; the growth of the amphiphilic aggregates makes these species more hydrophobic, with the consequent precipitation from the aqueous medium. The surfactant can increase the solubility interacting with 


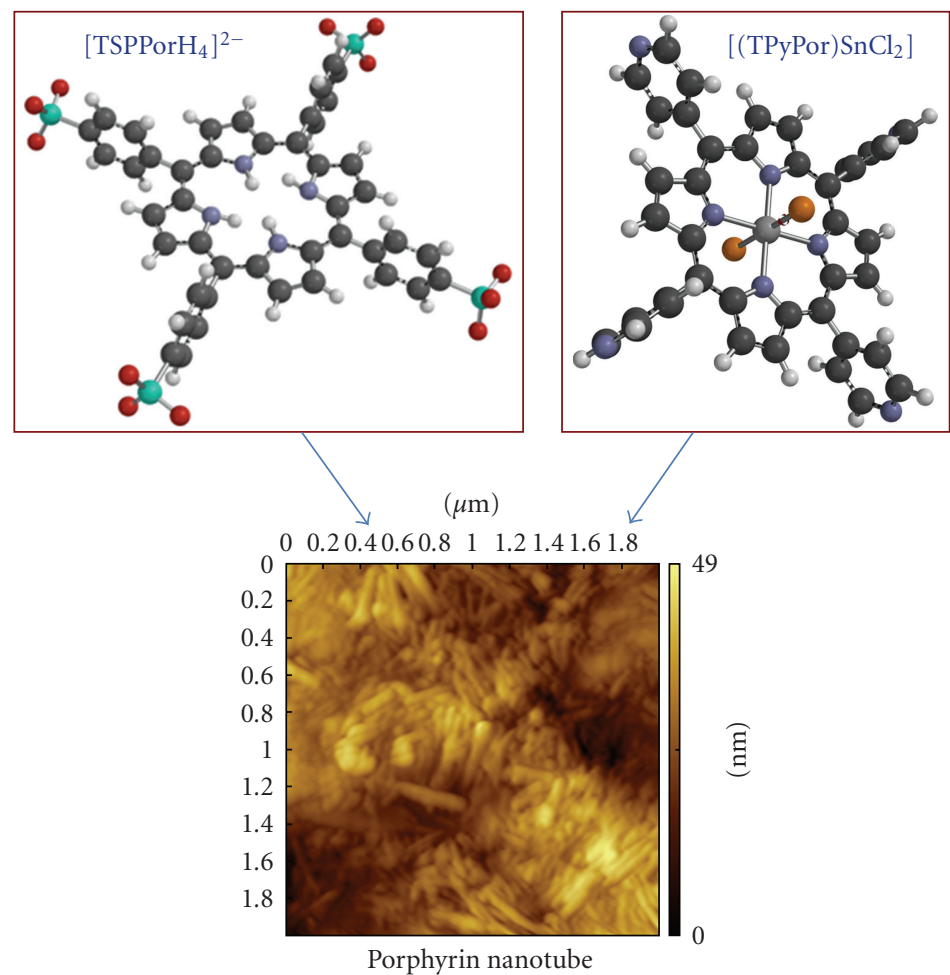

FIGURE 2: Molecular structures of the porphyrin moities and AFM image of the deposited porphyrin nanotubes.

the growing aggregates, allowing the formation of larger nanostructures.

2.5. Porphyrin Nanotubes. Porphyrin nanotubes have been obtained by aggregation of oppositely charged porphyrin units as reported by Wang et al. [26]. The ionic self-assembly of two porphyrins bearing opposite ionic charge leads to the formation of J-aggregates, whose nonplanar shape induce the formation of nanotubular structures, reaching free floating aggregates having lengths of tens of nanometers.

Variation of the porphyrin structures may strongly influence the nanostructure obtained by ionic self-assembly. Nanofibers, for example, can be obtained in the assembling of an oxoantimony complex of tetraphenylporphyrin (Figure 1(f)) with tetrasulfonatophenylporphyrin [27].

Porphyrin nanotubes were also obtained by self-assembly of an $\mathrm{Mo}(\mathrm{V})$ complex of the dodecaphenylporphyrin, a fully substituted derivative having a saddle distorted macrocycle (Figure 1(g) ) [28]. In this case the formation of the tubular structure takes advantage from the curved surface of the distorted porphyrin.

2.6. Miscellaneous of Porphyrin Aggregates. The first studies on the development of nanostructured porphyrin materials are related to the formation of the so-called "porphyrin sponges," that is, nanoporous solids having cavities of molecular sizes [29]. These supramolecular networks were obtained by self-assembling driven by interactions of very weak nature and several molecules of solvates were necessary to stabilize the porphyrin assemblies. Robust porphyrin-based organic zeolite analogs were prepared by self-assembling of TPyP metal complexes; these threedimensional frameworks showed high termal stability and a size-selective sorption mechanism towards guest species [30]. Following the same concept, $\mathrm{H}_{2}$ TCPP macrocycle has also been used as building block for the prepration of porphyrin-based zeolite analogs. More robust frameworks were obtained from the self-assembling of resorcinolsubstituted porphyrins; peripheral hydrogen bonds were exploited for the construction of supramolecular ordered solids, where the inner cavities can be exploited as selective molecular recognition sites. Kojima et al. studied the potentialities of bis- and tetraresorcinol substituted porphyrins as selective sensing layers using Quartz Crystal Microbalance technique [31].

Porphyrin nanochannels have been recently reported by Harada and Kojima [32], obtained by the self-assembly of the dodecaphenylporphyrin dihydrochloride salt during crystallization from $\mathrm{CHCl}_{3} / \mathrm{CH}_{3} \mathrm{CN}$ [33]. The saddle distortion of the macrocycle leads to the formation of porphyrin nanochannel where guest molecules can be included into the cavities. Porphyrin dication framework acts as electron deficient host where electron rich molecules, such as, for example, hydroquinone derivatives, are clathrate as guests.

We reported a facile and straightforward way to obtain porphyrin film with ordered morphology by spontaneous deposition of aggregates of amphiphilic tetraphenylporphyrin derivatives, possessing a cationic appended functionality [34]. The deposition of porphyrin aggregates is driven by hydrophobic effects, and, noteworthy, occurs spontaneously only in the case of the formation of ordered and 


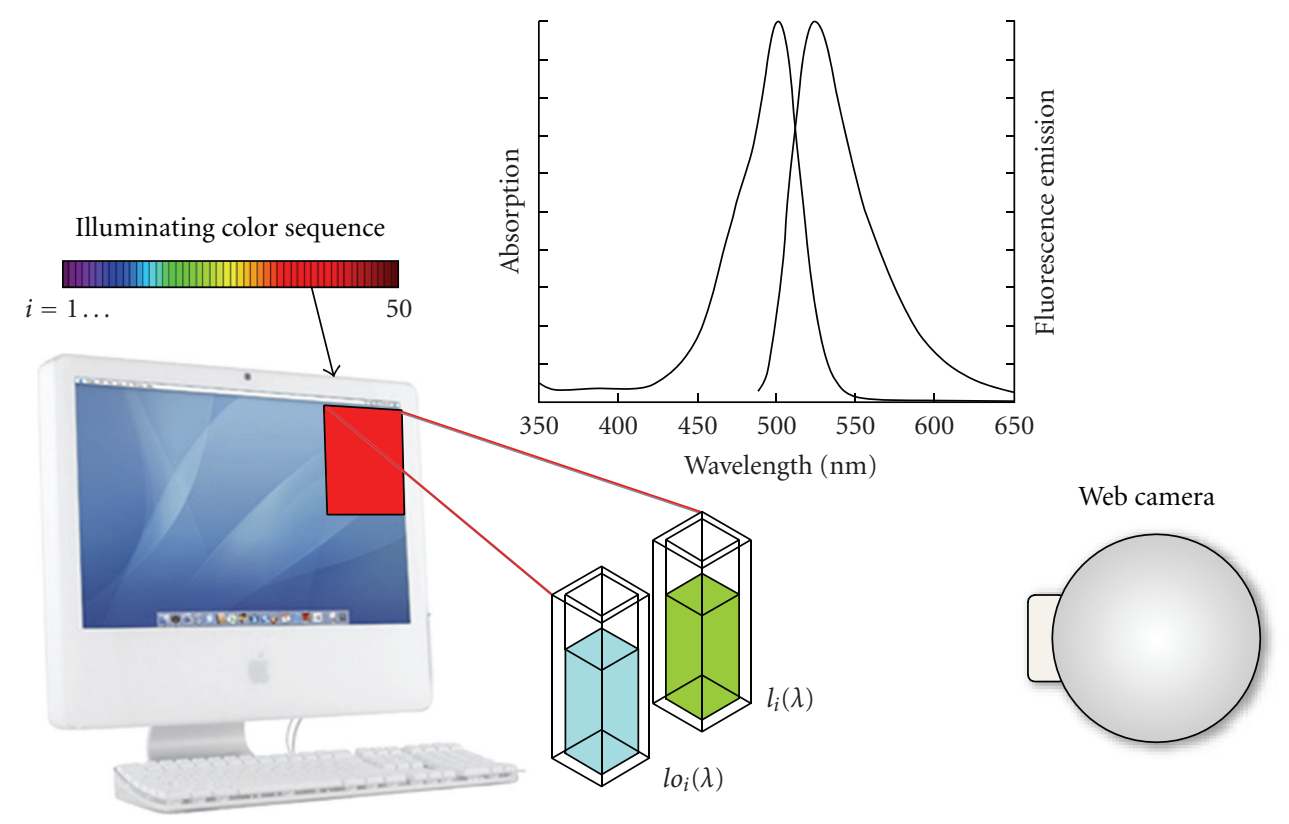

FIGURE 3: Sketch of the CSPT platform.

uniform aggregates. The occurrence of $\pi$-cation interaction should be taken into consideration for the achievement of these structures with ordered morphology. Deposition of the relative manganese or cobalt derivatives analogously occurs and the UV-visible spectral pattern of the films dramatically changes upon exposure to vapours of amines or olefins. The exploitation of these films for the construction of chemical sensors is reported in the following section.

\section{Chemical Sensor Applications of Porphyrin Nanostructures}

It is worth mentioning that while a great effort has been devoted to the preparation and characterization of porphyrin nanostructured materials, quite surprisingly only scant examples of practical applications have been reported focused in particular on catalysis or photovoltaic devices [7].

3.1. Porphyrin Nanotubes in CSPT Platform. Since chemical sensors could represent one of the most promising application fields for these porphyrin nanostructures, we have been interested in the exploitation of the porphyrin nanotubes, prepared by Wang's method [26], as sensing materials [35] (Figure 2). This geometrical motif is particularly appealing for sensor applications, because it can potentially show a dramatic increase in sensitivity/selectivity performances to respect the individual subunits. For example, the presence of the inner cavity can give raise to selective endohedral inclusion of different guests, or the interaction with analytes can alter the supramolecular arrangement of the molecular nanostructure; both these interaction pathways, alter the porphyrin J-aggregate optical properties.

Optical transduction is for this reason the mechanism of choice for such a nanostructure. The interaction mechanism

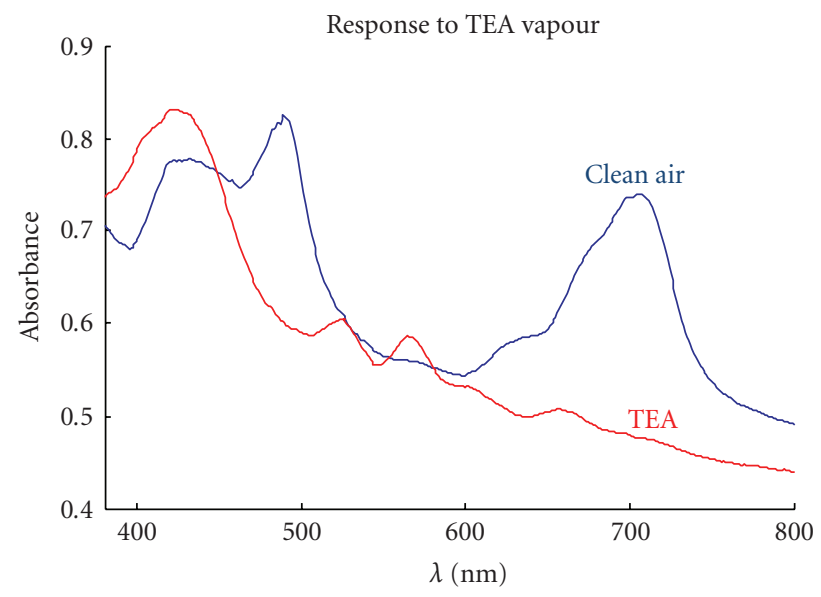

FIGURE 4: Nanotube optical spectra before and after the addition of $10^{-3} \mathrm{~mol} / \mathrm{L}$ of triethylamine in solution.

of porphyrin nanotubes with analytes in aqueous solutions has been firstly investigated; subsequently, the development of solid state chemical sensors through their deposition in thin films has been attempted.

As sensing platform we decided to use the Computer Screen Photoassisted Technology (CPST); based on the combination of a computer screen, programmed to display millions of color combining wavelengths in the optical range, and a digital camera, decomposing the light into three broad channels, this technique is able to perform a spectroscopy measurement allowing a simultaneous evaluation of absorbance and fluorescence of samples (Figure 3) [36-38].

We first studied the modification of absorption spectra induced by the addition of different salts to solutions of both precursor porphyrins and formed nanotubes. We noted that 


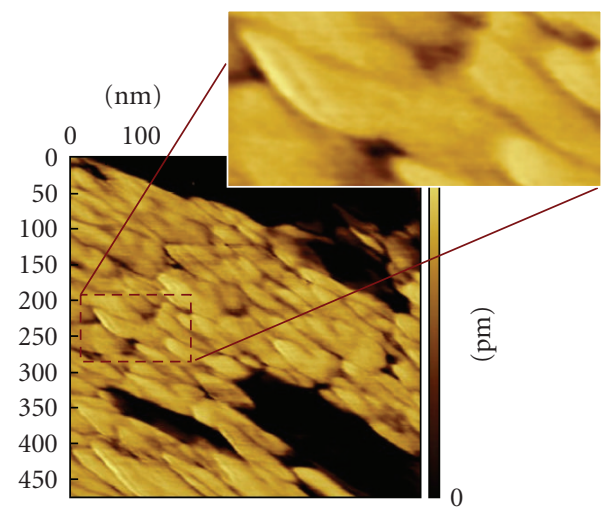

(a)

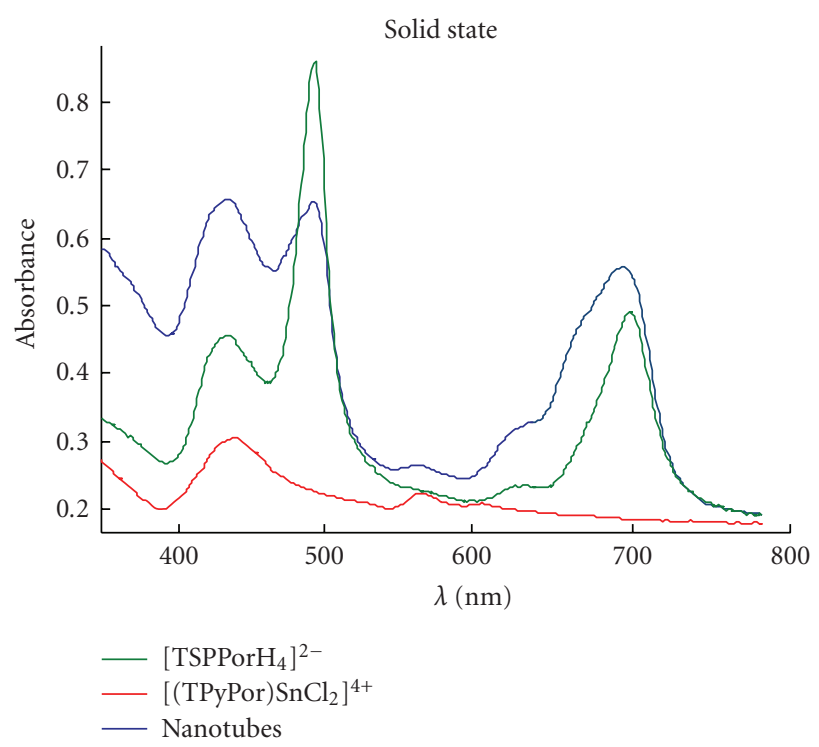

(b)

Figure 5: (a) AFM phase contrast image of a nanotube layer. In the inset a close-up view of a single structure is visible; (b) optical spectra of the porphyrin moities and porphyrin nanotube in the solid state.

porphyrin nanotubes could offer additional features for ions detection, which cannot be possible in the case of isolated porphyrin moieties.

With these encouraging results, we have then studied the variations induced by the addition of organic compounds dissolved in solution, such as triethylamine (Figure 4), where it should be noted that other interaction mechanisms, such as acid-base or even coordination for the $\mathrm{SnTPyPCl} \mathrm{C}_{2}$ moiety could be involved. Also in this case the porphyrin nanostructure was more sensitive than the subunits to the analyte addition.

This behavior prompted us to explore the possibility to utilize the chemical sensitivity of porphyrin nanotubes to detect compounds in gaseous phase. The subsequent step was devoted to the deposition of porphyrin nanotubes as solid layers to be used in the CSPT optical sensing platform. This is a critical step, because the forces involved are low energy interactions and consequently the resulting nanostructures are not rigid and they can be easily deformed when transferred in the solid film. Atomic force microscopy image of a layer of nanotubes directly deposited from the aqueous solution suggested the retention of the tubular structures, although their flattening cannot be excluded (Figure 5).

The direct deposition of nanotubes resulted in a film characterized by an excessive optical density that was not adequate to be measured with the CSPT. In order to overcome this problem nanotubes were dispersed in a polymeric matrix compatible with the formation process of the nanostructures. Polydimethylsiloxane (PDMS) was found compatible and the embedding of nanostructures inside the polymer took place as evidenced by optical spectra. Also in this case we cannot exclude a flattening of the porphyrin nanotubes into the polymeric matrix, but the retention of the aggregate features in the resulting optical spectra allowed anyway their exploitation for sensing purpose. In order to study the sensing properties of nanotubes-polymer mixture PDMS was also requested not to interfere with the sensing process; as a matter of fact the chosen polymeric matrix showed a good permeability to the investigated compounds.

The response of sensing layer upon exposure of volatiles was measured by the differential absorbance, obtained subtracting the absorbance measured in pure nitrogen atmosphere as background.

As shown from CSPT signature, the sensing mechanism of the porphyrin nanotubes probably does not involve the inner cavity, with the analytes inducing a modulation of the interaction between porphyrins keeping the nanostructure in shape; nonetheless the differences between the changes of CSPT absorbance in presence of model volatiles suggest that a manifold of interactions is likely to take place (Figure 6).

The exploitation of the porphyrin aggregates is not limited to gases or volatiles detection, but these nanostructured assemblies can also be used in the solution.

3.2. Amphiphilic Porphyrin Aggregates. We have been involved in the development of chemical sensor based on amphiphilic porphyrin aggregates. We observed spontaneous deposition from an aqueous solution $\left(\mathrm{H}_{2} \mathrm{O} / \mathrm{EtOH} 9: 1 ; \nu: v\right)$ of the amphiphilic porphyrin derivative reported in Figure 7 on silanised glass surfaces; the solid film, resulting from a controlled J-type aggregation fashion, presents, beside a good mechanical stability, an appreciable fluorescence emission [39].

This property can be a crucial feature in the sensing of heavy and transition metal ions that typically quench the luminescence, often via deactivation to the triplet state by heavy metal atom effect. On the basis of these 


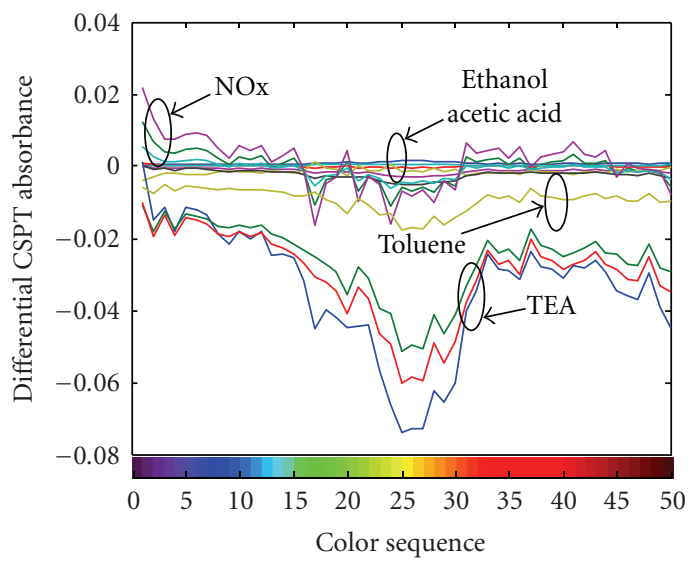

(a)

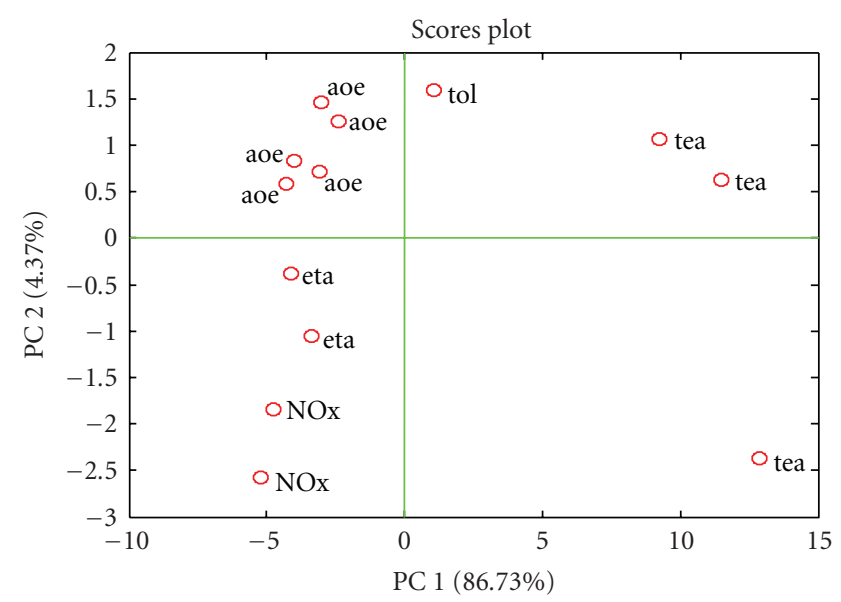

(b)

Figure 6: (a) Differential CSPT absorbances measured exposing the nanotube layers to the different vapors and gases. (b) Plot of the first two principal components calculated from the fingerprints shown in (a). Label data points indicate the compounds: tea: triethylamine, NOx, eta: ethanol, ace: acetic acid, and tol: toluene.

interesting results we investigated the potentialities of these simple systems for the construction of an efficient and specific luminescent solid-state chemosensor for the selective detection of $\mathrm{Hg}^{2+}$ ions in water.

We observed that the porphyrin emission was strongly quenched by simply dipping the porphyrin layered glass slides into diluted solutions of heavy metal salts, as reported in Figure 8: the efficient quenching of fluorescence emission of layered porphyrins by simple immersion into a solution of $\mathrm{Hg}^{2+} 1.0 \times 10^{-5} \mathrm{M}$ (Figure 8, trace d) is highlighted. Moreover the initial intensity could be restored, without loss of efficiency, washing the glass slides with a solution of $N, N, N^{\prime}, N^{\prime}$-tetrakis(2-pyridilmethyl) ethylenediammine (TPEN).

A consistent quenching was also observed in the case of $\mathrm{Hg}^{2+}$ activity, as low as $1 \mu \mathrm{M}$ concentration, pointing out the appreciable sensitivity of the investigated system. The concomitant examination of the UV-vis spectral pattern changes revealed the occurrence of a bathochromic shift of the porphyrin chromophores upon metal ion interaction, indicating the onset of coordinative interactions between the porphyrin core and the mercuric ion.

To test the reversibility and the stability of the system in operative conditions OFF/ON cycles (i.e., quenching with $\mathrm{Hg}^{2+}$, restoring in a TPEN solution) were also performed. The results are graphically reported in Figure 9 and show that a cycle can be repeated several times, with no appreciable loss of sensitivity, or dissolution of the film. Moreover the authors extended this protocol to a flow-through device.

To study this issue, a porphyrin coated flow-through quartz cell was used and the fluorescence emission responses, upon passage of $\mathrm{Hg}^{2+}$ solutions, analyzed. Also in this case, a quenching of fluorescence was observed, when a solution of $\mathrm{Hg}^{2+}$ passed through the cell. The fluorescence change was found to be dependent on the ion concentration.

More interestingly a good selectivity of the porphyrin layer responses with respect to other transition and posttran- sition metal ions, such as $\mathrm{Cd}^{2+}, \mathrm{Pb}^{2+}$, and $\mathrm{Cu}^{2+}$ was observed. Appreciable quenching of fluorescence, in fact, resulted only in the presence of more concentrated solution ( $\mathrm{mM}$ range) of the corresponding ions, as shown in Figure 10.

Competition experiments revealed negligible interferences by the other metal cations. The spectroscopic changes featured in the presence of $\mathrm{Hg}^{2+} 1.0 \times 10^{-5} \mathrm{M}$ remained unaffected by further addition of $\mathrm{Cd}^{2+}, \mathrm{Cu}^{2+}$, and $\mathrm{Pb}^{2+}$ up to $100 \mu \mathrm{M}$.

Conversely, a quenching effect could be observed upon addition of $\mathrm{Hg}^{2+}(10 \mu \mathrm{M})$ to a still emissing solution of the other cations $(\geq 100 \mu \mathrm{M})$. The inspection of the UVvis spectra upon addition of cations other than $\mathrm{Hg}^{2+}$ did not reveal evident perturbation of the porphyrin electronic states. The Soret band remained, in fact, virtually unchanged even at high concentration of added $\mathrm{Cd}, \mathrm{Cu}$, or $\mathrm{Pb}$ cations. This finding safely rules out, in these latter cases, the occurrence of metal coordination.

3.3. Towards Chiral Recognition. Following this approach, we surmised that the presence of a cationic chiral functionality on the porphyrin periphery would result in the achievement of solid-state systems expressing elements of supramolecular chirality. For this reason we synthesized a tetraphenylporphyrin bearing an (L)-prolininium moiety at one of the peripheral phenyl groups; this cationic chiral functionality steers the self-aggregation process toward the formation of large porphyrin aggregates featuring high supramolecular chirality, as evidenced by their CD spectra [40].

Prompted by these results we decided to perform some detailed spectroscopic studies (UV-Visible, CD, and RLS) on the heteroaggregation of amphiphilic chiral cationic porphyrin derivatives carried out in the presence of chiral aggregates of the anionic, that is, negatively charged chiral counterpart. The molecular structures of the porphyrin derivatives used in these studies are reported in Figures 7(b)$7(\mathrm{c})[40]$. 


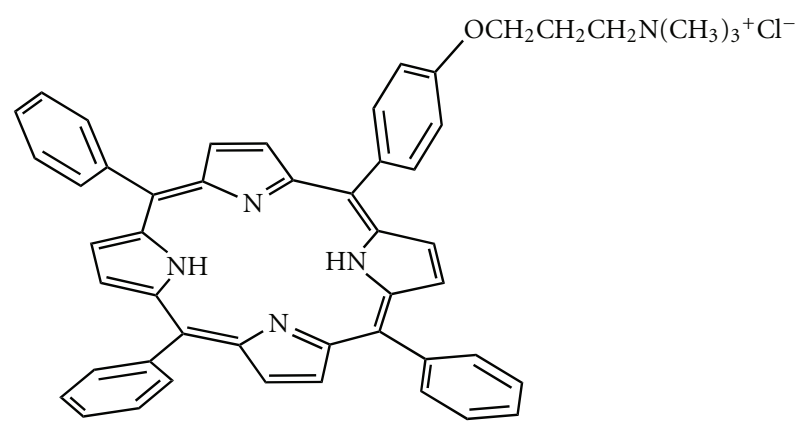

(a)<smiles></smiles>

(b)

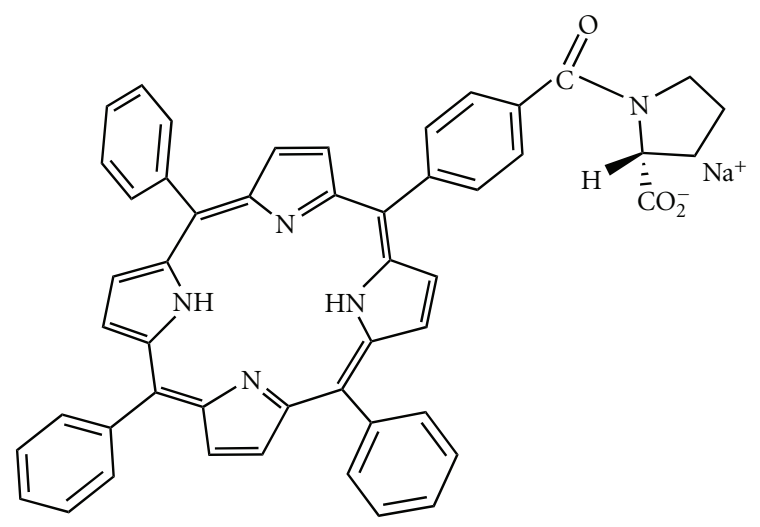

(c)

FIGURE 7: Molecular structures of (a) cationic amphiphilic porphyrin; (b) chiral cationic and anionic; (c) porphyrin counterparts.

Interesting features are evidenced by these binary systems. If the self-aggregation of the cationic moiety is carried out in the presence of preformed chiral aggregates of the anionic counterpart, the aggregation still occurs in a chiral fashion as witnessed by CD spectral changes upon heteroaggregation and more insight, a dramatic amplification of the resulting supramolecular aggregates, of about twenty-fold, is observed (Figure 11).

The ellipticity of the resulting heteroaggregates is higher compared to the one of the virtual sum " $a+b$ " aggregates. This should indicate a strong template effect exerted by

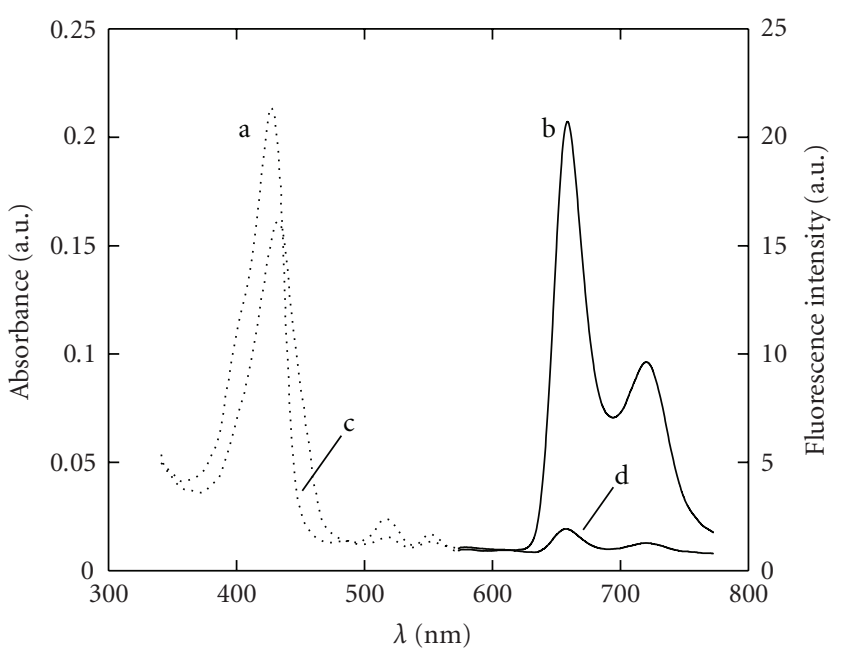

FIgURE 8: Absorption (dashed lines) and emission (solid lines, $\lambda$ ex $420 \mathrm{~nm}, \lambda \mathrm{em} 654,720 \mathrm{~nm}$ ) of porphyrin layers at different $\mathrm{Hg}^{2+}$ concentrations. (a) and (b) $\left[\mathrm{Hg}^{2+}\right]=0.0 \mathrm{M}$; (c) and (d) $\left[\mathrm{Hg}^{2+}\right]=$ $1.0 \times 10^{-5} \mathrm{M}$.

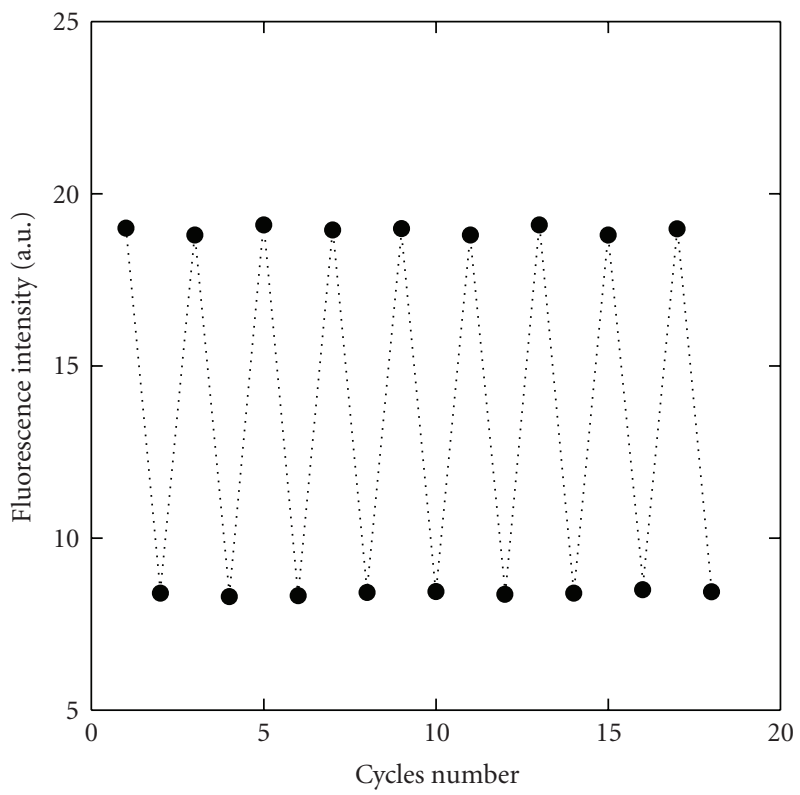

FIGURE 9: ON/OFF cycles of porphyrin layers in different aqueous solutions.

the presence of the anionic assemblies, reasonably onset by electrostatic forces.

The results obtained show that the electrostatic-templated aggregation induces a remarkable amplification of the chirality of the final porphyrin suprastructure. This can be of importance, interalia, for the construction of complex hetero-porphyrin architectures in which the supramolecular chirality can be tuned ad hoc.

In summary, the results obtained in the case of the templated aggregation open interesting perspectives for the achievement of new porphyrin-based supramolecular 


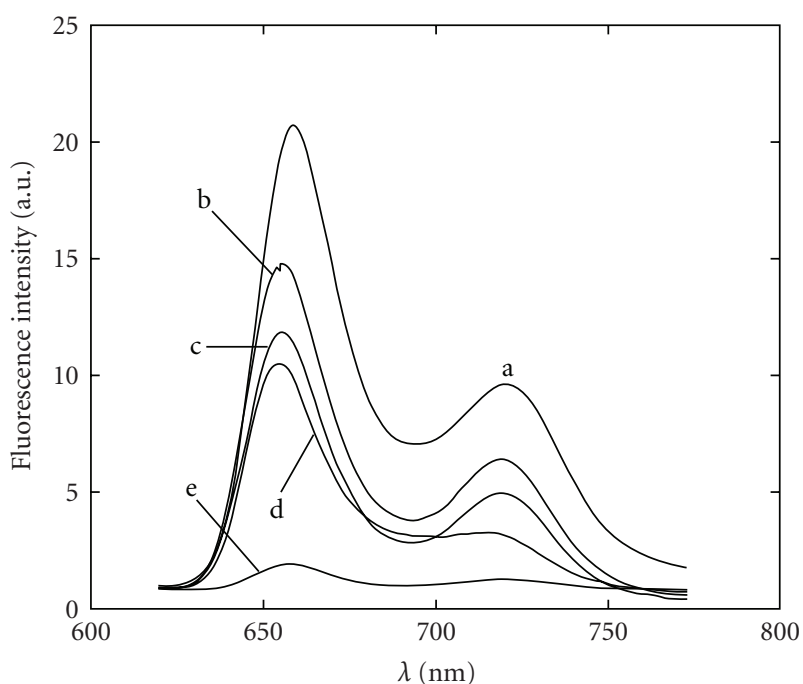

FIGURE 10: Fluorescence emission ( $\lambda$ ex $420 \mathrm{~nm}$ ) of porphyrin layers in different solutions. (a) $\mathrm{H}_{2} \mathrm{O}$, (b) $\mathrm{Cd}^{2+}$ (saturated solution), (c) $\mathrm{Cu}^{2+}\left(1.0 \times 10^{-3} \mathrm{M}\right),(\mathrm{d}) \mathrm{Pb}^{2+}\left(1.0 \times 10^{-3} \mathrm{M}\right)$ and $(\mathrm{e}) \mathrm{Hg}^{2+}(1.0 \times$ $\left.10^{-5} \mathrm{M}\right)$.

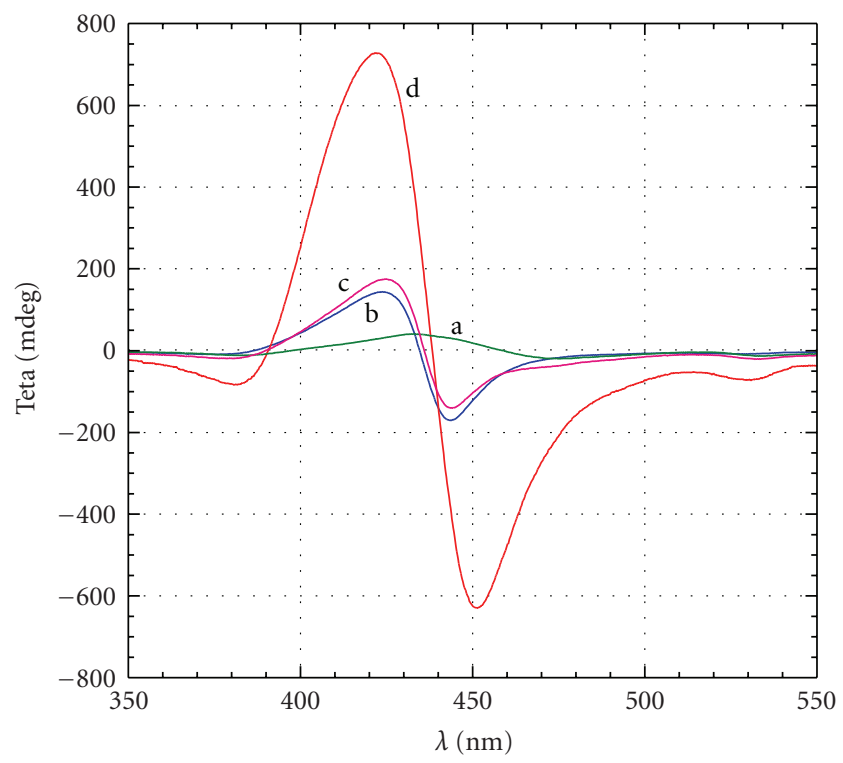
a) $\mathrm{PL}(+)$ aggregates $\left(7.5 \times 10^{-6} \mathrm{M}\right)$
b) $\operatorname{PL}(-)$ aggregates $\left(1.5 \times 10^{-5} \mathrm{M}\right)$
c) Virtual " $a+b$ " aggregates
d) Templated aggregation

Figure 11: Amplification of CD signal (line d) for templated aggregation compared to both individual chiral aggregates (lines (a and b) and their virtual sum (line c).

species bearing attractive properties for the construction of stereoselective sensor systems, and the development of such a sensors is currently undergoing in our laboratories.

\section{Conclusions}

The synthetic versatility of porphyrins allows the preparation of molecular units capable of assembling in ordered supramolecular structures, characterized by different geometrical motifs. While porphyrin nanostructures have offered an elegant and rational approach to study the way to prepare supramolecular networks, trying to learn and mimic the lesson given by biological systems, the study of these systems is now reaching the exploitation in functional devices. Seminal applications of porphyrin nanostructures in sensor devices show their promising potentialities, where the supramolecular network can offer additional interaction pathways, exalting the selectivity/sensitivity performances, which cannot be offered by the single molecular unit. The possibility to prepare chiral porphyrin nanostructures opens the way to the deposition of layers suited for chiral discrimination; their successful exploitation in chemical sensors would have a tremendous impact in all the fields where chirality plays a fundamental role.

\section{References}

[1] G. M. Whitesides, "Nanoscience, nanotechnology, and chemistry," Small, vol. 1, no. 2, pp. 172-179, 2005.

[2] J. V. Barth, G. Costantini, and K. Kern, "Engineering atomic and molecular nanostructures at surfaces," Nature, vol. 437, no. 7059, pp. 671-679, 2005.

[3] S. Iijima, "Helical microtubules of graphitic carbon," Nature, vol. 354, no. 6348, pp. 56-58, 1991.

[4] S. V. N. T. Kuchibhatla, A. S. Karakoti, and S. Seal, "Hierarchical assembly of inorganic nanostructure building blocks to octahedral superstructures-a true template-free selfassembly," Nanotechnology, vol. 18, no. 7, Article ID 075303, 4 pages, 2007.

[5] J. M. Lehn, in Supramolecular Chemistry. Concepts and Perspectives, VCH, Weinheim, Germany, 1995.

[6] J.-M. Lehn, "Supramolecular chemistry-scope and perspectives: molecules, supermolecules, molecular devices (Nobel Lecture)," Angewandte Chemie International Edition, vol. 27, no. 1, pp. 89-112, 1988.

[7] A. C. Grimsdale and K. Müllen, "The chemistry of organic materials," Angewandte Chemie International Edition, vol. 44, pp. 5592-5629, 2005.

[8] T. S. Balaban, "Tailoring porphyrins and chlorins for selfassembly in biomimetic artificial antenna systems," Accounts of Chemical Research, vol. 38, no. 8, pp. 612-623, 2005.

[9] K. M. Kadish, K. M. Smith, and R. Guilard, Eds., The Porphyrin Handbook, vol. 1, Academic Press, San Diego, Calif, USA, 2000.

[10] S. Horn, K. Dahms, and M. O. Senge, "Synthetic transformations of porphyrins-advances 2004-2007," Journal of Porphyrins and Phthalocyanines, vol. 12, no. 10, pp. 10531077, 2008.

[11] K. M. Kadish, K. M. Smith, and R. Guilard, Eds., The Porphyrin Handbook, vol. 6, Academic Press, San Diego, Calif, USA, 2000.

[12] K. M. Kadish, K. M. Smith, and R. Guilard, Eds., The Porphyrin Handbook, vol. 4, Academic Press, San Diego, Calif, USA, 2000. 
[13] X. Gong, T. Milic, C. Xu, J. D. Batteas, and C. M. Drain, "Preparation and characterization of porphyrin nanoparticles," Journal of the American Chemical Society, vol. 124, no. 48, pp. 14290-14291, 2002.

[14] C. M. Drain, G. Smeureanu, S. Patel, X. Gong, J. Garno, and J. Arijeloye, "Porphyrin nanoparticles as supramolecular systems," New Journal of Chemistry, vol. 30, no. 12, pp. 1834$1843,2006$.

[15] A. S. D. Sandanayaka, Y. Araki, T. Wada, and T. Hasobe, "Structural and photophysical properties of self-assembled porphyrin nanoassemblies organized by ethylene glycol derivatives," Journal of Physical Chemistry C, vol. 112, no. 49, pp. 19209-19216, 2008.

[16] A. K. Perepogu and P. R. Bangal, "Preparation and characterization of free-standing pure porphyrin nanoparticles," Journal of Chemical Sciences, vol. 120, no. 5, pp. 485-491, 2008.

[17] Z. Wang, Z. Li, C. J. Medforth, and J. A. Shelnutt, "Selfassembly and self-metallization of porphyrin nanosheets," Journal of the American Chemical Society, vol. 129, no. 9, pp. 2440-2441, 2007.

[18] T. N. Milic, N. Chi, D. G. Yablon, G. W. Flynn, J. D. Batteas, and C. M. Drain, "Controlled hierarchical self-assembly and deposition of nanoscale photonic materials," Angewandte Chemie International Edition, vol. 41, no. 12, pp. 2117-2119, 2002.

[19] A. D. Schwab, D. E. Smith, C. S. Rich, E. R. Young, W. F. Smith, and J. C. de Paula, "Porphyrin nanorods," Journal of Physical Chemistry B, vol. 107, no. 41, pp. 11339-11345, 2003.

[20] A. D. Schwab, D. E. Smith, B. Bond-Watts, et al., "Photoconductivity of self-assembled porphyrin nanorods," Nano Letters, vol. 4, no. 7, pp. 1261-1265, 2004.

[21] S. C. Doan, S. Shanmugham, D. E. Aston, and J. L. McHale, "Counterion dependent dye aggregates: nanorods and nanorings of tetra(p-carboxyphenyl)porphyrin," Journal of the American Chemical Society, vol. 127, no. 16, pp. 5885$5892,2005$.

[22] M. C. Lensen, K. Takazawa, J. A. A. W. Elemans, et al., "Aided self-assembly of porphyrin nanoaggregates into ring-shaped architectures," Chemistry, vol. 10, no. 4, pp. 831-839, 2004.

[23] T. Hasobe, H. Oki, A. S. D. Sandanayaka, and H. Murata, "Sonication-assisted supramolecular nanorods of mesodiaryl-substituted porphyrins," Chemical Communications, no. 6, pp. 724-726, 2008.

[24] T. Hasobe, A. S. D. Sandanayaka, T. Wada, and Y. Araki, "Fullerene-encapsulated porphyrin hexagonal nanorods. An anisotropic donor-acceptor composite for efficient photoinduced electron transfer and light energy conversion," Chemical Communications, no. 29, pp. 3372-3374, 2008.

[25] S. J. Lee, J. T. Hupp, and S. T. Nguyen, "Growth of narrowly dispersed porphyrin nanowires and their hierarchical assembly into macroscopic columns," Journal of the American Chemical Society, vol. 130, no. 30, pp. 9632-9633, 2008.

[26] Z. Wang, C. J. Medforth, and J. A. Shelnutt, "Porphyrin nanotubes by ionic self-assembly," Journal of the American Chemical Society, vol. 126, no. 49, pp. 15954-15955, 2004.

[27] Z. Wang, K. J. Ho, C. J. Medforth, and J. A. Shelnutt, "Porphyrin nanofiber bundles from phase-transfer ionic self-assembly and their photocatalytic self-metallization," Advanced Materials, vol. 18, no. 19, pp. 2557-2560, 2006.

[28] T. Kojima, R. Harada, T. Nakanishi, K. Kaneko, and S. Fukuzumi, "Porphyrin nanotubes based on self-assembly of $\mathrm{Mo}(\mathrm{V})$-dodecaphenylporphyrin complexes and inclusion of Mo-oxo clusters: synthesis and characterization by $\mathrm{X}$ ray crystallography and transmission electron microscopy," Chemistry of Materials, vol. 19, no. 1, pp. 51-58, 2007.
[29] J.-H. Chou, M. E. Kosal, H. S. Nalwa, N. A. Rakow, and K. S. Suslick, "Applications of porphyrins and metalloporphyrins to materials chemistry," in The Porphyrin Handbook, K. M. Kadish, K. M. Smith, and R. Guilard, Eds., vol. 4, pp. 43-131, Academic Press, San Diego, Calif, USA, 2000.

[30] K. Ariga, K. Endo, Y. Aoyama, and Y. Okahata, "QCM analyses on adsorption of gaseous guests to cast films of porphyrinresorcinol derivatives," Colloids and Surfaces A, vol. 169, no. 1-3, pp. 177-186, 2000.

[31] T. Kojima, T. Nakanishi, T. Honda, and S. Fkuzumi, "Photoinduced electron transfer in supramolecular assemblies involving saddle-distorted porphyrins and phthalocyanines," Journal of Porphyrins and Phthalocyanines, vol. 13, pp. 14-21, 2009.

[32] R. Harada and T. Kojima, "A porphyrin nanochannel: formation of cationic channels by a protonated saddle-distorted porphyrin and its inclusion behavior," Chemical Communications, pp. 716-718, 2005.

[33] D. Monti, M. Venanzi, M. Russo, et al., "Spontaneous deposition of amphiphilised porphyrin ordered film on glass," New Journal of Chemistry, vol. 28, pp. 1123-1128, 2004.

[34] F. Dini, E. Martinelli, G. Pomarico, et al., "Chemical sensitivity of self-assembled porphyrin nano-aggregates," Nanotechnology, vol. 20, no. 5, Article ID 055502, 8 pages, 2009.

[35] D. Filippini, S. P. S. Svensson, and I. Lundström, "Computer screen as a programmable light source for visible absorption characterization of (bio)chemical assays," Chemical Communications, vol. 9, no. 2, pp. 240-241, 2003.

[36] D. Filippini, A. Alimelli, C. Di Natale, R. Paolesse, A. D’Amico, and I. Lundström, "Chemical senses of familiar devices," Angewandte Chemie International Edition, vol. 45, pp. 38003803, 2006.

[37] E. Gatto, M. A. Malik, C. Di Natale, et al., "Polychromatic fingerprinting of excitation emission matrices," Chemistry- A European Journal, vol. 14, no. 20, pp. 6057-6060, 2008.

[38] L. S. Dolci, E. Marzocchi, M. Montalti, et al., "Amphiphilic porphyrin film on glass as a simple and selective solid-state chemosensor for aqueous $\mathrm{Hg}^{2+}$," Biosensors and Bioelectronics, vol. 22, no. 3, pp. 399-404, 2006.

[39] D. Monti, M. Venanzi, G. Mancini, C. Di Natale, and R. Paolesse, "Supramolecular chirality control by solvent changes. Solvodichroic effect on chiral porphyrin aggregation," Chemical Communications, no. 19, pp. 2471-2473, 2005.

[40] D. Monti, M. Venanzi, M. Stefanelli, et al., "Chiral amplification of chiral porphyrin derivatives by templated heteroaggregation," Journal of the American Chemical Society, vol. 129, no. 21, pp. 6688-6689, 2007. 

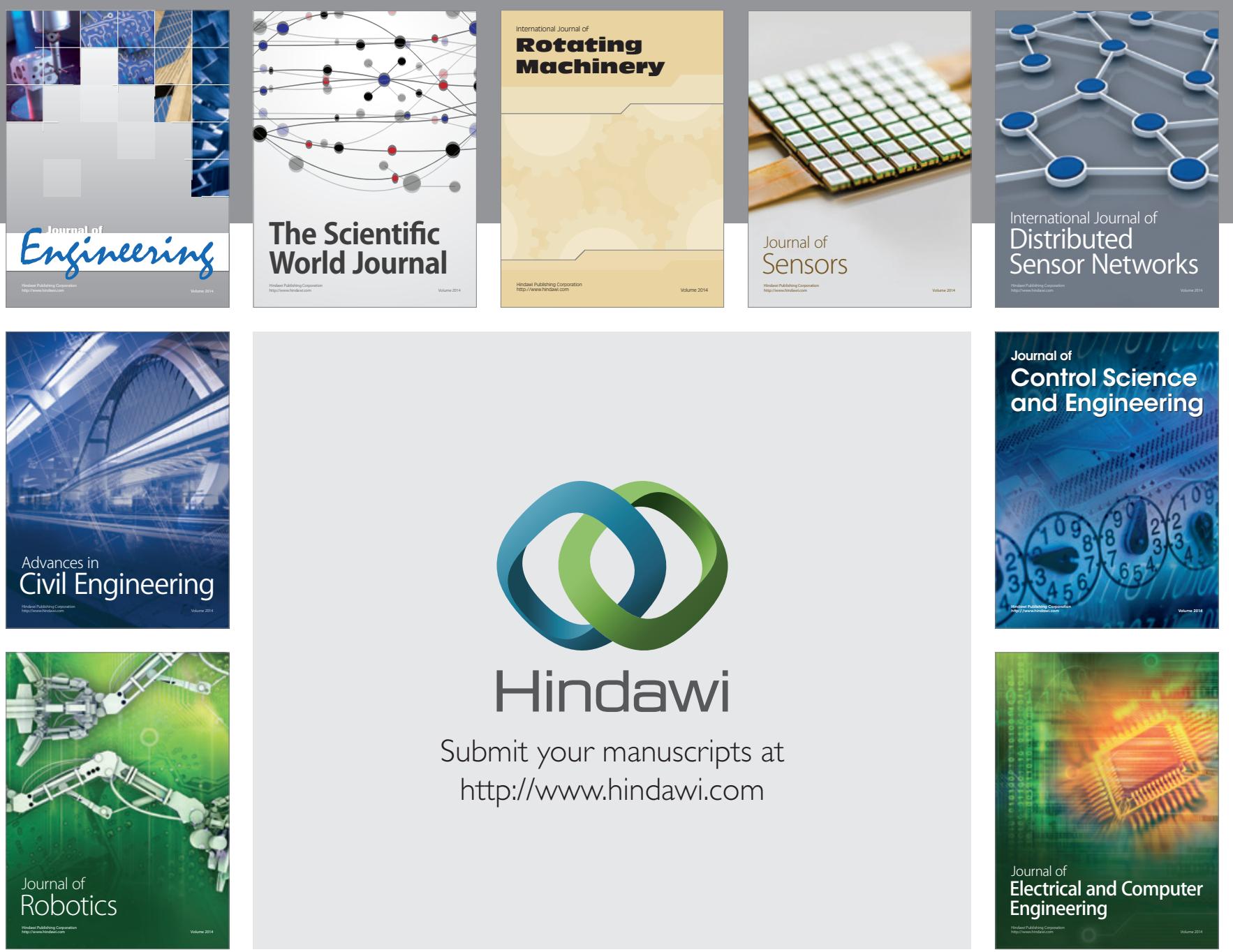

Submit your manuscripts at

http://www.hindawi.com
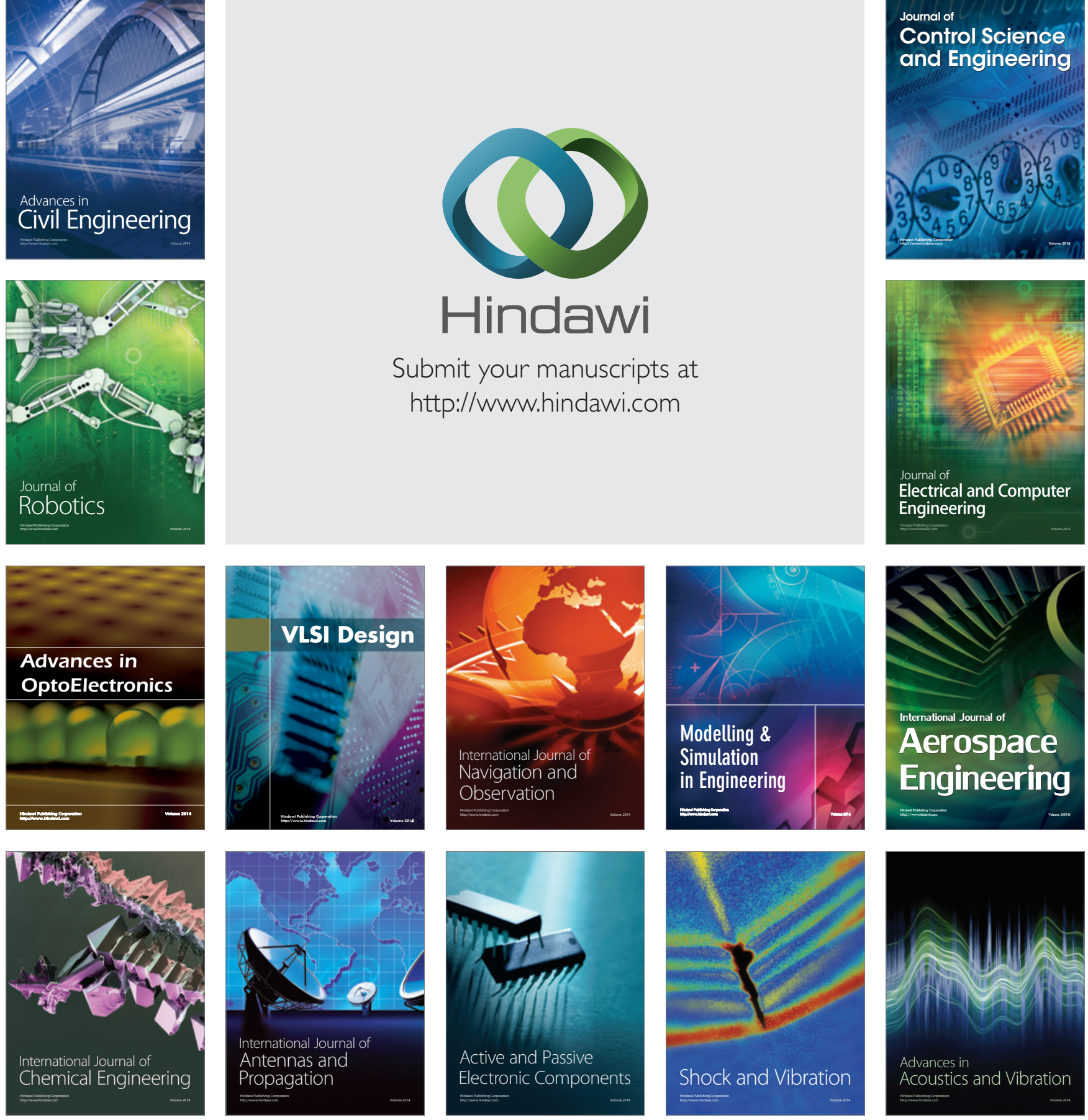